Cahiers $d u$ MONDE RUSSE

\section{Cahiers du monde russe}

Russie - Empire russe - Union soviétique et États indépendants

$46 / 4 \mid 2005$

L'invention d'une politique humanitaire

\title{
Ju. N. Afanas'ev et al., Istorija stalinskogo GULAGa
}

Marta Craveri et Marc Elie

\section{OpenEdition \\ Journals}

Édition électronique

URL : https://journals.openedition.org/monderusse/6601

DOI : 10.4000/monderusse.6601

ISSN : $1777-5388$

Éditeur

Éditions de l'EHESS

Édition imprimée

Date de publication : 1 décembre 2005

Pagination : 905-915

ISBN : 2-7132-2057-2

ISSN : $1252-6576$

Référence électronique

Marta Craveri et Marc Elie, «Ju. N. Afanas'ev et al., Istorija stalinskogo GULAGa », Cahiers du monde russe [En ligne], 46/4 | 2005, mis en ligne le 06 juillet 2009, consulté le 03 septembre 2022. URL

http://journals.openedition.org/monderusse/6601 ; DOI : https://doi.org/10.4000/monderusse.6601

Ce document a été généré automatiquement le 3 septembre 2022.

Tous droits réservés 


\title{
Ju. N. Afanas'ev et al., Istorija stalinskogo GULAGa
}

\author{
Marta Craveri et Marc Elie
}

\section{RÉFÉRENCE}

Istorija stalinskogo GULAGa : konec 1920-h - pervaja polovina 1950-h godov. Sbornik dokumentov v semi tomah/The History of Stalin's GULAG : Late 1920sEarly 1950s. Collected Documents in 7 Volumes. Redakcionnyj sovet izdanija : Ju. N. AFANAS'EV et al. Predsedatel' : V. P. KOZLOV. Moscou : ROSSPEN, 2004-2005

1 Le projet d'une Histoire du Goulag stalinien, mis en œuvre par les Archives d'État de la Fédération de Russie (GARF) et la Hoover Institution on War, Revolution and Peace, a enfin vu le jour après quasiment dix ans de travaux. L'ensemble des six volumes thématiques, auxquels a été adjoint postérieurement un volume d'inventaire des fonds dépouillés, constitue une tentative d'exposition encyclopédique du Goulag de 1930 à 1953. Par « Goulag », les auteurs n'entendent pas seulement l'Administration centrale des camps (GULag), mais aussi l'ensemble de la politique répressive et de ses conséquences sociales et économiques sous Stalin. Cette collection est unique en son genre. Par son ampleur, elle prend naturellement une place centrale dans l'historiographie du Goulag et est appelée à devenir la référence documentaire pour toute étude à venir sur le système carcéral soviétique.

Chaque volume traite d'un aspect particulier du régime pénitentiaire en URSS et est dirigé par des spécialistes différents; il mérite donc d'être évalué séparément. Avant de passer à l'examen de chacun des tomes, il convient de signaler deux principes que les éditeurs ont adoptés pour l'ensemble de la collection. Si le choix d'écrire l'histoire du Goulag sous Stalin semble de lui-même déterminer les bornes chronologiques de l'entreprise (1930-1953), la plupart des auteurs se sont sentis à l'étroit dans ce cadre et ont introduit des documents qui portent sur la période qui suit la mort de Stalin, voire sur celle qui précède son accession au pouvoir. Car, après tout, s'il s'agit bien de découvrir le 
Goulag au sens large, pourquoi s'en être tenu aux deux décennies les mieux connues? Autre choix discutable : les éditeurs ont fortement limité la place des notes explicatives dans chaque volume. Ainsi, les documents administratifs restent opaques, ce qui limite l'impact du projet aux seuls spécialistes. Cela correspond à la tendance générale dans l'édition de documents depuis l'ouverture des archives en Russie, mais on aurait pu espérer qu'une entreprise aussi ambitieuse aurait fait exception.

T. 1 : Massovye repressii v SSSR [Les répressions de masse en URSS], 2004, 728 p.

Otv. red. : S. V. Mironenko, N. Werth ; otv. sost. : I. A. Zjuzina

3 Ce volume ouvre brillamment la série. Il couvre toutes les formes de répression de 1930 à 1953 et comporte également un chapitre sur la sortie du stalinisme, de la mort du dictateur au XXe Congrès du parti en 1956. Les auteurs ont découpé chronologiquement l'ouvrage en sept sections, chacune comprenant plusieurs subdivisions qui rassemblent des documents sur une campagne répressive donnée : "passeportisation ", déportations des "peuples punis", lutte contre le vol, etc. La première section couvre les années 1930-1932, centrées sur la dékoulakisation; la seconde aborde la terreur pendant la famine dans les années 1932-1934; la troisième est axée sur la période 1933-1936 qui marque une accalmie dans la répression; la quatrième traite en détail de la Grande Terreur; la cinquième, des répressions pendant la guerre ; la sixième, de la législation extraordinaire d'après-guerre, dont l'épisode moins connu de l'assaut contre les kolkhoziens «fainéants » en 1948 ; et enfin, la septième et dernière évoque en quelques documents la révision de la politique répressive, depuis la mort de Stalin jusqu'au milieu des années 1950.

Cette organisation des documents autour des campagnes de répression donne à voir le rythme particulier des accès de violence punitive sous Stalin, déjà mis en évidence dans l'historiographie (par exemple par Peter Solomon ${ }^{1}$ ). Les auteurs éclairent les phases de reflux qui suivent les paroxysmes répressifs : l'amnistie des victimes et la condamnation des excès commis par polices, tribunaux et pouvoirs locaux faisaient ainsi partie intégrante du processus. Le recul de la répression suite à la famine de 1932-1933 et le « retour à la légalité socialiste » après le summum de la "Grande Terreur » sont des cas bien connus. Le recueil montre un recul comparable lors de la campagne de durcissement de la discipline du travail au début de la guerre. Ainsi, aux condamnations massives des retardataires et des tire-au-flanc (2,5 millions en neuf mois pour la seule loi du 26 juin 1940), des petits vols sur le lieu de travail et des actes bénins de hooliganisme, accompagnées de mesures très coercitives à l'encontre des chefs d'entreprise laxistes et des magistrats libéraux, succède la libération d'une partie d'entre eux, immédiatement après le début de l'attaque allemande. D'autres seront libérés par l'amnistie de 1945.

Dans leur sélection documentaire, les auteurs ont donné la priorité aux directives ministérielles concernant l'application des décisions répressives et aux rapports des exécutants à leurs supérieurs. Beaucoup des documents normatifs les plus célèbres n'ont pas été reproduits : leur contenu est cité pour mémoire en note. C'est le cas par exemple de l'oukase de déportation des Allemands de 1941, des lois sur le vol de 1932 et 1947 ainsi que sur la discipline du travail de 1940-1941. D'autres étaient connus et cités : ils sont néanmoins publiés ici dans leur intégralité.

6 Les annexes révèlent l'innovation majeure du volume : les auteurs y ont rassemblé la plus importante collection de statistiques pénales sur les répressions staliniennes publiée à ce jour. Ces tableaux ont tous été produits par l'administration soviétique après 1953, au moment où, désirant fixer les nouvelles orientations, les héritiers de Stalin ont 
commandé aux divers départements statistiques de tirer le bilan des années de frénésie pénale. Ce matériau brut réclame un important travail de synthèse et d'interprétation. Le lecteur est en effet plongé au cœur d'une jungle touffue, avec des dizaines de services administratifs, judiciaires et policiers, tous dotés de services statistiques distincts et souvent concurrents : tribunaux (civils réguliers, du transport, militaires, des camps...), troïkas, conférences spéciales, Collège militaire de la Cour suprême. Les chiffres se recoupent et paraissent incompatibles. On est loin de l'image naïve d'un tableau unique des victimes du stalinisme, mais ce chaos administratif en lui-même est une caractéristique de la répression sous Stalin.

7 Nicolas Werth a précédé ce volume clair, didactique même, d'une introduction qui expose les grandes divisions de l'ouvrage. Le texte est tiré du chapitre qu'il avait consacré à l'URSS dans l'ouvrage collectif Le livre noir du communisme $e^{2}$ sous le titre « Un État contre son peuple ». Le lecteur y trouvera d'une part un rappel utile des campagnes répressives et d'autre part une explication lumineuse des phénomènes considérés (voir le passage sur la Grande Terreur par exemple); c'est une aide minimale, indispensable à la compréhension des textes, étant donné l'avarice des notes de fin de volume. Synthèse documentaire d'années de recherches sur la violence d'État sous Stalin, ce volume est celui qui intéressera le public le plus large.

T. 2 : Karatel'naja sistema : struktura i kadry[Le système punitif : structure et cadres], 2004, $695 \mathrm{p}$.

Otv. red. : N. V. Petrov ; otv. sost. : N. I. Vladimircev

8 Ce volume est destiné aux spécialistes du Goulag au sens institutionnel du mot. Les documents rassemblés concernent deux domaines bien distincts: 1-la structure réglementaire des camps (textes relatifs à l'ouverture, la modification ou la fermeture des camps, aux zones de déportation, chantiers, etc.) ; 2 -tout ce qui se rapporte au personnel des camps (nombre, situation matérielle, recrutement, formation et carrière, promotions et sanctions). Il s'agit essentiellement de textes normatifs (ordres, instructions, circulaires).

9 On peut regretter que les auteurs aient opté pour un découpage chronologique très général et trop rigide du volume. Ils mélangent les deux thèmes - structure et cadres du Goulag - sans les distinguer, même par des sous-chapitres. Ce choix se fait au profit du premier et au détriment du second. Le plan du volume correspond ainsi aux évolutions de la structure du Goulag et néglige les cadres (gardiens, chefs de camps, bureaucrates de l'administration centrale). Certains documents exceptionnels mériteraient pourtant d'être mis en valeur, comme cette note du procureur général à Stalin (mai 1934) qui fait le point sur les crimes commis par les gardiens aux Solovki et dans les camps du Nord (document $n^{\circ} 29$ ).

10 Les auteurs ont repris l'ordonnancement chronologique classique proposé par M. B. Smirnov, S. P. Sigačev et D. V. Škapov dans leur introduction à l'ouvrage consacré au système des camps de redressement par le travail en URSS ${ }^{3}$. Une première partie est consacrée aux années 1930. Les documents y évoquent le processus qui a marqué l'évolution des lieux de détention soviétiques vers le Goulag stalinien. Soit comment l'OGPU - police politique, qui fusionne en 1934 avec le commissariat du peuple aux Affaires intérieures (NKVD) - en est venue à dominer tout le système carcéral soviétique. C'est à l'OGPU que le Bureau politique fixe en 1929 la tâche de mettre en œuvre des projets de colonisation en faisant un large usage du travail des détenus et en développant ses propres lieux de détention, qui finiront par avaler ceux du commissariat du peuple à 
la Justice. C'est l'OGPU qui est chargée de centraliser toutes les administrations pénitentiaires sous un ministère unique. Enfin, c'est à l'OGPU-NKVD que la direction politique confie les projets d'industrialisation les plus ambitieux.

11 La période suivante - qui va du début de la guerre à la mort de Stalin - est celle de l'épanouissement du système pénitentiaire stalinien, véritable «complexe carcéroproductif». La guerre voit la multiplication des formes de détention (camps spéciaux pour prisonniers de guerre ou speclagerja, camps de filtration, sections de bagne). Les documents sur l'après-guerre montrent l'extension du réseau des camps qui annexe des secteurs toujours plus larges de l'économie. On se félicite ici de l'attention que les auteurs portent aux camps spéciaux (osobye lagerja), jetant les bases d'une histoire de cette forme pénitentiaire, souvent évoquée mais mal connue. Une trentaine de documents, presque tous inédits, racontent comment ces camps ont pris une place centrale dans le système carcéral dès leur création en février 1948. Dans une dernière partie, qui couvre les années 1953 et 1954, les auteurs publient une vingtaine de documents qui témoignent du début de décomposition qui atteint le système du Goulag immédiatement après la mort de son créateur. Pour compléter cette fresque historique, les auteurs ont pu faire une exception au cadre chronologique fixé pour l'ensemble de la collection en ajoutant en fin de volume une centaine de pages consacrées aux dix premières années du régime. L'intérêt historique d'un tel ajout est évident.

12 L'initié, toujours satisfait de disposer de documents nouveaux, saluera cet ensemble touffu. Le novice, lui, sera rebuté, car il est difficile de se repérer dans ce volume. Il faut dire que le lecteur est abandonné face à des textes administratifs souvent ardus. L'introduction n'apporte aucune clarté. La trame événementielle de l'histoire de l'Administration centrale des camps est développée de manière trop dense, alors que la question des personnels est là encore assez délaissée. Pas de grands espoirs à placer dans l'appareil de notes, qui n'est abondant dans aucun des tomes de la collection, comme on l'a dit, mais qui est réduit ici à 25 pages seulement. On notera que la liste des abréviations est incomplète. En revanche, les notices biographiques sont utiles car, même très brèves, elles fournissent des informations sur la carrière des principaux responsables des camps.

T. 3 : Ekonomika Gulaga [L'économie du Goulag], 2004, 624 p.

Otv. red. i sost. : O. V. Hlevnjuk

13 Ce volume, dirigé par Oleg Khlevniuk, est divisé en deux grands chapitres thématiques. Dans le premier, on trouve, par ordre chronologique, des documents (ordres, directives, comptes rendus, bilans) qui montrent les différentes phases de l'organisation et du développement du travail forcé, ainsi que les nombreux problèmes qui émergent au fur et à mesure de l'utilisation de plus en plus massive d'une main-d'œuvre forcée dans différents secteurs de l'économie soviétique. Le second est consacré aux objectifs et aux branches principales de cette économie forcée. On y découvre des documents relatifs à la construction de grands chantiers et de bassins industriels : le canal Belomor reliant la mer Blanche à la mer Baltique, le trust Uhto-Pečorskij, le Dal'stroj, la ligne de chemin de fer Baïkal-Amour (BAM), le canal Moscou-Volga, le complexe industriel de nickel de Norilsk, les chantiers et entreprises militaires chargés du projet nucléaire soviétique (Glavpromstroj et Première direction principale), le canal Volga-Don, les centrales hydroélectriques de Kuibyshev et de Stalingrad, les exploitations forestières et les industries du bois, les camps agricoles et de produits de consommation. Ces textes apportent des informations 
sur la genèse des projets d'exploitation de certaines régions, sur les principaux obstacles rencontrés et sur la façon de les surmonter.

La plus grande partie des documents publiés ici sont conservés dans différents fonds des Archives d'État de la Fédération de Russie (GARF) : le fonds du NKVD-MVD, le fonds du Sovnarkom et du Conseil des ministres et le fonds "Goulag ». On trouve aussi de nombreux matériaux issus des différentes administrations économiques des camps créés en 1941 ainsi que des dossiers spéciaux (osobaja papka) que la direction du NKVD/MVD envoyait régulièrement à Stalin, Molotov, Hruščev, Voznesenskij et à d'autres dirigeants. Enfin neuf documents très intéressants sur l'activité économique de l'OGPU dans les années 1930 viennent des Archives du FSB. L'ensemble permet de mieux comprendre les mécanismes décisionnels relatifs aux questions économiques du Goulag.

De nombreux documents datent des années 1930, période où se forme la structure économique du Goulag, tel qu'il va fonctionner et se développer jusque dans la première moitié des années 1950. Ces sources montrent que, dans les débuts, les perspectives de développement du Goulag n'apparaissaient pas clairement aux yeux des dirigeants ; c'est la construction du canal Belomor qui s'avère déterminante pour la future organisation économique de l'OGPU et de l'Administration centrale des camps. Tout un ensemble de matériaux témoignent de la crise que connurent les camps à partir de 1938 aussi bien au niveau de la production que de l'organisation, en tant que conséquence directe de la Grande Terreur: plans inachevés, qualité du travail catastrophique, chute de la production économique. D'autres montrent comment Berija va reprendre la situation en main, et créer, en janvier 1939, le Bureau technique du NKVD pour être à même d'exploiter au mieux les prisonniers sur la base de connaissances technologiques.

Les documents des années 1941-1945 permettent de suivre l'évolution du rôle du Goulag dans l'économie de guerre et la place importante qu'il occupera grâce à sa réserve de main-d'œuvre, à un moment où tous les autres ministères économiques en sont déficitaires. Très intéressants enfin sont ceux qui couvrent la période de l'après-guerre : on y retrouve toutes les tentatives pour relancer production et productivité, notamment par le biais de l'introduction de salaires et la décision de libérer de manière anticipée certains prisonniers pour les garder comme salariés sur place.

7 Ce volume contient les documents les moins connus de l'histoire du Goulag - une histoire exclusivement économique, mais où la suprématie du politique, sur laquelle Khlevniuk insiste depuis des années, émerge dans toutes les discussions, préoccupations et décisions du leadership sur le développement économique du travail forcé.

T. 4 : Naselenie Gulaga : Čislennost' $\mathbf{i}$ uslovija soderžanija [La population du Goulag : effectifs et conditions de détention], 2004, 624 p.

Otv. red. : A. B. Bezborodov, sost. : V. M. Hrustalev et I. V. Bezborodova

18 Ce volume est l'un des plus riches et des plus originaux de la série. Pour la première fois, un recueil d'archives donne toute sa place à la vie quotidienne des détenus et de leurs gardiens. Tout le monde des camps avec ses horreurs est révélé aux yeux du lecteur et vient confirmer ce qui était déjà connu grâce aux mémoires d'anciens détenus.

Les auteurs ont divisé ce tome en quatre chapitres correspondant chacun grosso modo à un service de l'Administration centrale des camps. Le premier rassemble les documents statistiques sur les détenus (nombre, origine sociale, etc.) qui proviennent pour l'essentiel du Département de statistique et de répartition des détenus (OURZ). C'est là un ensemble considérable, qui complète et permet d'affiner ce qui avait déjà été publié, en 
particulier dans le recueil GULAG, 1918-19604. Eu égard au souci encyclopédique de la collection, on regrette que les auteurs n'aient pas prolongé ces statistiques par régions et par républiques pour l'après-guerre. Il est dommage aussi qu'ils n'aient pas expliqué et commenté en note le formulaire statistique standard de l'Administration des camps, que l'on retrouve dans une demi-douzaine de documents sur toute la période $\left(\mathrm{n}^{\circ} 18,33\right.$ et 51-54) et qui est incompréhensible ${ }^{5}$.

Le second chapitre couvre le régime de détention, sur la base surtout des documents du Département des opérations (Operativnyj otdel). Il éclaire des phénomènes mal connus comme le droit accordé à certains prisonniers de se déplacer sans convoi entre zones d'habitation et de travail, et même en dehors de ces zones pour les besoins de la production (raskonvojrovanie). Certaines sources traitent entre autres de la censure exercée sur le courrier des prisonniers, des procédures d'examen des plaintes, du droit de visite, de l'introduction progressive d'un système de salaires. L'utilisation de détenus pour les besoins personnels des chefs de camp, en particulier comme domestiques, fait l'objet d'un document très informatif ( $\left.n^{\circ} 159\right)$.

21 L'alimentation et l'approvisionnement des détenus - dont était chargé le Département $\mathrm{du}$ ravitaillement du Goulag - fait l'objet d'un troisième chapitre. Les auteurs publient ici les instructions concernant les normes alimentaires des détenus pour toute la période. Celles-ci étaient considérées par l'administration comme le stimulus le plus important de la productivité du travail. Des documents mettent au jour les combines de l'administration pour déposséder les prisonniers de ce qu'elle leur devait selon les normes, pourtant très faibles pour toute la période considérée. Le lecteur se souvient des descriptions des grands mémorialistes du Goulag : le pain, qui était la nourriture de base, et souvent la seule, était ainsi manipulé de différentes manières pour en augmenter le poids sans utiliser de farine (cuisson trop courte, addition de clous, de savon etc., cf. document $n^{\circ} 232$ ). Certains textes décrivent la mise en place après la guerre de soussections sanitaires chargées du rétablissement des détenus les plus affaiblis, d'un système de vente au détail et d'une organisation de fournitures vestimentaires. Enfin, le chapitre qui clôt le volume décrit comment le Département sanitaire suivait de près la morbidité et la mortalité des prisonniers.

22 L'introduction de Bezborodova ne donne pas un tableau historique cohérent des conditions de détention des détenus ni de leur vie quotidienne, mais elle met en lumière quelques tendances essentielles, comme la dépendance hiérarchique des services médicaux des camps par rapport au NKVD dans les années 1930 (et non par rapport au ministère de la Santé). L'auteur énonce néanmoins des jugements de valeur, ou des points de vue, qui mériteraient des éclaircissements: qu'est-ce que «le faible niveau moral » des employés du Goulag ? Le rétablissement de l'économie après la guerre, qui aurait fait sentir ses effets positifs dans les années 1950, explique-t-il vraiment l'amélioration des conditions d'existence dans les camps après la mort de Stalin? En quoi précisément le Goulag a-t-il influencé la langue, la culture et les mœurs?

23 Si Bezborodova montre bien la confusion des statistiques, la difficulté de les comparer dans le temps et le flou des catégories, et si elle engage une discussion intéressante avec A. I. Kokurin et Ju. N. Morukov, elle n'approfondit cependant pas suffisamment cette question; celle-ci reste donc un point important de dissension historiographique qui attend toujours une étude exhaustive. Elle reprend même des fables anciennes à l'encontre des chiffres qu'elle cite : il convient de rappeler que ce n'est pas le $\mathrm{XX}^{\mathrm{e}}$ Congrès du parti qui a libéré 580000 détenus politiques; l'écrasante majorité d'entre eux sont 
sortis des camps entre 1953 et fin 1955, avant le « Rapport secret » et les Commissions de libération d'avril 1956, en tirant leur peine jusqu'au bout, ou encore de manière anticipée, grâce aux crédits des journées travaillées (tableau n ${ }^{\circ} 1$, p. 39 ).

T. 5 : Specpereselency v SSSR [Les déplacés spéciaux en URSS], 2004, 823 p.

Otv. red. i sost. : T. V. Carevskaja-Djakina

Les documents de ce volume sont divisés en cinq chapitres chronologiques et thématiques: liquidation des koulaks en tant que classe, 1930-1935; déportations ethniques et politiques pendant la Grande Terreur et au début de la Deuxième Guerre mondiale, 1935-1941; fonctionnement du système des déplacés spéciaux pendant la guerre, 1941-1943; changements juridiques et administratifs en rapport avec les déportations ethniques et politiques, 1943-1947 ; crise du système des déplacés spéciaux, 1948-1953.

L'ensemble du recueil est composé de textes juridiques, circulaires et ordres des différents organes du pouvoir concernés par la répression des déplacés spéciaux (Sovnarkom, OGPU, NKVD/MVD, Goulag et Parquet), ainsi que de rapports et comptes rendus de la Section des déplacés spéciaux du Goulag pour les différentes régions de déportation. Les statistiques ont une place très limitée car elles ont déjà été amplement publiées dans le passé par Viktor Zemskov. Le choix des documents permet d'observer la constitution progressive de la gigantesque structure administrative chargée des différentes catégories de déplacés spéciaux, déportés spéciaux et colons de travail qui, entre 1930 et 1950, ont été déportés dans l'Oural, en Sibérie et dans les républiques d'Asie centrale (surtout au Kazakhstan).

Le volume retrace toute la politique de répression stalinienne, nettement ciblée sur un ensemble large, mais très ponctuel, de catégories de population jugées indésirables, "socialement étrangères", suspectes, nuisibles, ou qui auraient pu constituer une " cinquième colonne" d'ennemis susceptible de frapper en cas de conflit. Ces répressions s'effectuent par vagues successives. Les premières victimes sont les paysans qui s'opposent prétendument à la collectivisation : 1800000 sont déportés entre 1930 et 1931 et constituent jusqu'en 1940 la majorité des déplacés spéciaux. Vient ensuite le tour des catégories dites du "désordre social », c'est-à-dire tous ceux qui, pour des raisons non politiques, ne s'adaptent pas au nouvel ordre soviétique: délinquants, " hooligans ", mendiants, vagabonds (tziganes inclus) et prostituées, qui sont déportés lors des épurations des grandes villes. Puis les nationalités potentiellement ennemies font l'objet de toute une série de mesures répressives : diasporas, habitants des régions frontalières, émigrés politiques réfugiés en Union soviétique. Finlandais, Polonais, Lettons, Estoniens, Grecs, Roumains, Bulgares, Kurdes, Iraniens, Chinois, Coréens sont ainsi les victimes de ces opérations répressives, mais la déportation la plus importante dans cette catégorie touche la population d'origine allemande: près d'un million d'Allemands sont déportés à partir de 1941 en Sibérie et au Kazakhstan. L'invasion allemande marque un tournant dans la déportation des nationalités, qui constituèrent, avec les centaines de milliers de déplacés en provenance de l'Ukraine occidentale et des pays Baltes, la majorité des victimes pendant et après la guerre: Grecs, Finlandais, Arméniens, Kurdes, Polonais, peuples du Nord Caucase, Tatars de Crimée, etc.

Parallèlement aux documents sur l'organisation des différentes vagues répressives et sur leur gestion administrative, on trouve un important choix de textes en provenance des Directions du NKVD/MVD des régions de destination sur la situation des déplacés spéciaux: conditions de vie et de travail, situation sanitaire, taux de mortalité, 
problèmes liés aux évasions massives des lieux de déportation. Ces dernières prendront de telles proportions après la guerre qu'elles pousseront le Conseil des ministres à retirer la gestion des déplacés spéciaux au MVD pour la confier au MGB, plus performant dans le contrôle et la répression des infractions.

Cela donne un ensemble vivant : aux données juridiques et administratives s'ajoutent les aspects de la vie quotidienne des déplacés spéciaux, les mille problèmes auxquels ils sont confrontés et les différentes solutions envisagées par l'administration pour les résoudre.

Les années qui suivent la mort de Stalin sont négligées, comme dans l'ensemble des six volumes où les documents explicitant les différents enjeux liés à la sortie du système concentrationnaire sont rares. L'auteur a laissé de côté ici l'ensemble de la documentation, en partie déjà publiée, qui montre comment les différentes catégories de déplacés spéciaux ont été libérées et autorisées à rentrer dans leur territoire d'origine.

T. 6: Vosstanija, bunty i zabastovki zaključennyh [Révoltes, émeutes et grèves de prisonniers], 2004, $736 \mathrm{p}$.

Otv. red. i sost. : V. A. Kozlov, O. V. Lavinskaja

30 Le volume est divisé en quatre chapitres chronologiques : émeutes et grèves de la faim entre 1930 et 1940, rébellions pendant la guerre, désordres (1945-1953), enfin révoltes (surtout celles de Norilsk, Vorkuta et Kengir) après la mort de Stalin. Le choix de l'ordre chronologique permet d'observer une lente, mais constante augmentation de toutes les manifestations de résistance auxquelles vont être confrontées les administrations des camps.

31 Les documents concernant la première période donnent davantage d'informations sur les efforts de l'OGPU pour créer un réseau d'informateurs à l'intérieur des camps que sur de réelles menaces de la part des prisonniers, car les épisodes de résistance sont très rares. Pendant les années de guerre, en revanche, les organes de sécurité signalent une augmentation alarmante des tentatives de rébellion dans tous les camps. Parmi les causes principales, on trouve l'évolution de la population concentrationnaire pendant le conflit : départ d'une grande partie des prisonniers avec des peines allégées pour le front, concentration de prisonniers plus « dangereux » et déterminés, entrée dans les camps des élites militaires des pays Baltes et des territoires occidentaux de la Pologne occupés par l'Armée rouge à partir de 1939. Les organisations clandestines et la résistance des prisonniers ne font que croître, pour s'étendre dans tout l'Archipel, avec la politique répressive menée par le NKVD dans l'après-guerre. L'arrivée massive de nationalistes baltes et ukrainiens et de partisans polonais et biélorusses confronte en effet les administrations à des prisonniers déterminés à utiliser dans les camps leur expérience de la guerre et de la lutte souterraine.

32 Les documents les moins connus, qui auraient sans doute pu faire l'objet d'un chapitre séparé et que l'on aurait souhaités plus nombreux, concernent les désordres organisés par les criminels et leurs conflits internes au sein du camp. On pensait jusque-là que c'étaient surtout les prisonniers politiques qui s'étaient organisés contre l'administration, mais on découvre ici un monde criminel très actif et menaçant, même s'il est très divisé. Un document extraordinaire de décembre 1949, dans lequel la Direction du Goulag ordonne aux administrations locales des camps de cesser immédiatement d'utiliser les "chiennes" pour des tâches administratives, montre dans le détail comment ces administrations exploitaient les tensions entre " chiennes » et "vory v zakone », ainsi que les répercussions néfastes qui s'ensuivaient. Auparavant, on avait un écho de ces faits 
uniquement grâce aux mémoires ou aux témoignages oraux, mais on ignorait comment cela avait été perçu de l'intérieur.

Certains documents ajoutent des éléments nouveaux à la connaissance du phénomène de la résistance dans les camps. Ils montrent que, déjà en 1945, les nationalistes baltes et ukrainiens étaient identifiés comme des éléments dangereux à l'intérieur des camps et faisaient l'objet d'une surveillance spéciale et d'actions " prophylactiques ». Le lecteur apprend aussi que l'amnistie de 1945 a été à l'origine de toute une série de problèmes car si, d'une part, les libérations anticipées désorganisent le réseau d'informateurs, d'autre part, la déception des prisonniers qui en sont exclus va se traduire, comme pour l'amnistie de 1953, par des évasions, des grèves et des émeutes. Dans une lettre du procureur général à Malen'kov, on découvre que, face à l'ampleur des évasions, il est proposé d'utiliser l'armée pour maintenir l'ordre dans certains camps. Enfin deux documents attestent que même les colonies pour enfants ont été le théâtre de désordres.

Si un nombre incalculable d'émeutes, de grèves, de révoltes, d'évasions et de désordres de toutes sortes sont répertoriés, une place moins importante est accordée aux répercussions de ces événements sur le fonctionnement même des camps et sur les difficultés rencontrées par les autres administrations dépendant en partie de la production des prisonniers. On dispose désormais, grâce à ce volume, d'un tableau exhaustif du phénomène de la résistance au Goulag, mais de nombreuses questions sur la crise que ces événements ont déclenchée restent en suspens.

T. 7 : Sovetskaja repressivno-karatel'naja politika i penitenciarnaja sistema $\mathbf{v}$ materialah Gosudarstvennogo arhiva Rossijskoj Federacii : Annotirovannyj ukazatel' del = Soviet Repressive-Penal Policy and Penal System in the Holdings of the State Archives of the Russian Federation: Annotated Guide to the Files, 2005, $712 \mathrm{p}$.

Otv. red. : V. A. Kozlov, S. B. Mironenko ; sost. A. B. Dobrovskaja

La vraie surprise de la série se trouve dans le septième volume, qui fournit la liste des dossiers les plus importants du GARF concernant la politique répressive de l'État soviétique de 1918 à 1960. Inouï! Le chercheur peut tranquillement compulser chez lui les fonds du GARF, sans avoir à se déplacer à Moscou pour commander des inventaires gras et blafards. Les en-têtes des dossiers ont été habilement retravaillés pour révéler leur contenu précis et sortir de l'approximation des interminables « correspondances sur questions générales et organisationnelles ». C'est l'outil indispensable à tout historien intéressé par les circonvolutions de la politique répressive de la majeure partie de l'ère soviétique. Bien au-delà de la seule administration du GULag, il englobe le Goulag au sens large où l'entend l'introduction de la collection, à savoir toute la politique répressive des débuts de l'ère soviétique jusqu'à la fin des années 1950. Ce Goulag-ci inclut par exemple le sort des enfants abandonnés. Notons, sur un plan pratique, que le retard dans la parution de ce dernier volume offre un avantage de taille : il est vendu séparément des six autres.

36 Certains fonds sont entièrement décryptés pour la période : ceux du GULAG (1930-1960) au sens élargi (c'est-à-dire incluant les déportés spéciaux, les colonies pour enfants, etc.) ; et ceux de son prédécesseur, l'Administration principale des lieux de détention du NKVD de l'URSS (1922-1930). On trouve également des ensembles archivistiques essentiels pour comprendre la politique pénale en général, comme ceux du ministère de la Justice et de la Cour suprême de l'URSS, dont les inventaires anciennement secrets sont entièrement décryptés ici jusqu'au milieu des années 1950. En revanche, les auteurs ont dû faire un tri parmi les dossiers des fonds les plus vastes, comme ceux du Conseil des ministres et du 
Parquet de l'URSS. La part du lion revient au fonds du NKVD de la RSFSR (1917-1930), intégralement reproduit. Ainsi, les années qui vont de la fondation du régime au tournant stalinien sont très bien couvertes, avec même les fonds moins connus de la Croix-Rouge politique à Moscou (1918-1922) et du Tribunal suprême du Comité central exécutif de la RSFSR (pour les années 1917-1924).

Les auteurs ont aussi ajouté la liste exacte des dossiers microfilmés pour le projet Hoover : voilà qui est utile pour savoir ce qu'il faut aller chercher au GARF, ou bien ce à quoi on a accès plus facilement et plus confortablement aux États-Unis ou ailleurs, dans les bibliothèques qui ont fait l'acquisition des précieuses bobines. En revanche, le lecteur ne prêtera aucune attention à la bibliographie, qui n'est pas à la hauteur du projet : elle est bien trop courte, périmée et incomplète.

\section{NOTES}

1. P. H. Solomon, Soviet Criminal Justice under Stalin, Cambridge : Cambridge University Press, 1996.

2. Stéphane Courtois et al., dirs., Le livre noir du communisme : crime, terreur, répression, Paris : $\mathrm{R}$. Laffont, 1997.

3. M. B. Smirnov, dir., Sistema ispravitel'no-trudovyh lagerej v SSSR, 1923-1960: Spravočnik, Moscou : Zven'ja, 1998.

4. A. I. Kokurin, N. V. Petrov, GULAG : Glavnoe upravlenie lagerej : 1918-1960, Moscou : MFD, 2002.

5. Oleg Khlevniuk le déchiffre dans The History of the Gulag from Collectivization to the Great Terror, New Haven : Yale University Press, 2004. Voir aussi J. Arch Getty, Gábor T. Rittersporn et Viktor N. Zemskov, "Victims of the Soviet Penal System in the Pre-War Years : A First Approach on the Basis of Archival Evidence », The American Historical Review, 98 (4), octobre 1993, p. 1017-1049, ici 1048-1049. 